Draft Version November 3, 2018

Preprint typeset using $\mathrm{LAT}_{\mathrm{E}} \mathrm{X}$ style emulateapj v. 12/14/05

\title{
MEAN MOTION RESONANCES FROM PLANET-PLANET SCATTERING
}

\author{
Sean N. Raymond ${ }^{1}$, Rory Barnes ${ }^{2}$, Philip J. Armitage ${ }^{3}$, \& Noel Gorelick 4 \\ (Accepted Sept 18, 2008) \\ Draft version November 3, 2018
}

\begin{abstract}
Planet-planet scattering is the leading mechanism to explain the large eccentricities of the observed exoplanet population. However, scattering has not been considered important to the production of pairs of planets in mean motion resonances (MMRs). We present results from a large number of numerical simulations of dynamical instabilities in 3-planet systems. We show that MMRs arise naturally in about five percent of cases. The most common resonances we populate are the 2:1 and 3:1 MMRs, although a wide variety of MMRs can occur, including high-order MMRs (up to eleventh order). MMRs are generated preferentially in systems with uneven mass distributions: the smallest planet is typically ejected after a series of close encounters, leaving the remaining, more massive planets in resonance. The distribution of resonant planets is consistent with the phase-space density of resonant orbits, meaning that planets are randomly thrown into MMRs rather than being slowly pulled into them. It may be possible to distinguish between MMRs created by scattering vs. convergent migration in a gaseous disk by considering planetary mass ratios: resonant pairs of planets beyond $\sim 1 \mathrm{AU}$ with more massive outer planets are likely to have formed by scattering. In addition, scattering may be responsible for pairs of planets in high-order MMRs (3:1 and higher) that are not easily populated by migration. The frequency of MMRs from scattering is comparable to the expected survival rate of MMRs in turbulent disks. Thus, planet-planet scattering is likely to be a major contributor to the population of resonant planets.
\end{abstract}

Subject headings: planetary systems: formation - methods: n-body simulations

\section{INTRODUCTION}

The current sample of exoplanets exhibits several interesting dynamical features (Butler et al.2006): here we focus on two of these. First, there is a vast range of planetary eccentricities, from zero to $>0.9$, with a median of 0.2 ( 0.27 for planets past 0.1 AU that have not been affected by tides; Rasio et al. 1996; Jackson et al. 2008). Second, mean motion resonances (MMRs) in multiple planet systems appear to be relatively common (e.g., Marcy et al.2001). There are 31 currently-known multiple planet systems comprising 44 pairs of adjacent planets, of which ten $(23 \%)$ show some evidence of resonances (Table 1). However, the evidence for resonances is tentative for all but a few cases.

Dynamical instabilities in systems of two or more planets can explain the wide eccentricity distribution of exoplanets (Rasio \& Ford 1996; Weidenschilling \& Marzari 1996; Lin \& Ida 1997). Instabilities arise on timescales that are related to the planets' initial separation (Marzari \& Weidenschilling 2002; Chatterjee et al. 2008), and lead to close encounters between planets and subsequent ejections or mergers. In the aftermath of close encounters, the surviving planets can statistically reproduce the observed eccentricity distribution of exoplanets (Adams \& Laughlin 2003; Juric \& Tremaine 2008; Chatterjee et al. 2008)

\footnotetext{
${ }^{1}$ NASA Postdoctoral Program Fellow, Center for Astrophysics and Space Astronomy, University of Colorado, Boulder CO 80309; raymond@lasp.colorado.edu

${ }^{2}$ Lunar and Planetary Laboratory, University of Arizona, Tucson, AZ; rory@lpl.arizona.edu

3 JILA, University of Colorado, Boulder CO 80309. pja@jilau1.colorado.edu

${ }_{4}^{4}$ Google, Inc., 1600 Amphitheatre Parkway, Mountain View, CA 94043; gorelick@google.com
}

MMRs are thought to arise primarily from convergent migration in gaseous protoplanetary disks (Snellgrove et al.2001; Lee \& Peale 2002). Indeed, models show that capture in the 2:1 and 3:2 MMRs is a particularly common occurrence (Thommes 2005; Pierens \& Nelson 2008; Lee et al. 2008). However, MRI-derived turbulence can act to remove planets from resonance. Adams et al. (2008) estimate that only $1 \%$ of resonant systems should remain for a disk lifetime of $1 \mathrm{Myr}$.

In this paper we attempt to reconcile the planet-planet scattering scenario with the population of resonant exoplanets. We numerically investigate dynamical instabilities in systems of three planets located at $\sim 2-10$ AU with a variety of mass distributions. We find that MMRs are a common occurrence, arising in 5-10 percent of unstable systems. Our simulations populate a range of MMRs, including the 2:1, 3:2, 3:1, 4:1 and extending up to much higher-order (Table 2). MMRs are populated by scattering at random into stable regions; the density of resonant orbits (i.e., the fraction of phase space that undergoes resonant oscillations) is consistent with the scattered resonant systems. We propose several ways to discriminate between scattering and convergent migration as the source of exoplanet MMRs.

\section{METHODS}

Our simulations started with three planets randomly separated by $4-5$ mutual Hill radii $\left(R_{H, m}=0.5\left(a_{1}+\right.\right.$ $\left.a_{2}\right)\left(\left[M_{1}+M_{2}\right] / 3 M_{\star}\right)^{1 / 3}$, where $a$ is the semimajor axis and $M$ the mass). This spacing was chosen to produce instabilities on timescales of at least the $\sim 10^{5}$ year timescale of runaway gas accretion ${ }^{5}$ (Pollack et

${ }^{5}$ Indeed, instabilities occurred on timescales from 100 years to $98 \mathrm{Myr}$ with a median of a few $\times 10^{5}$ years. In addition, about $1 / 4$ 


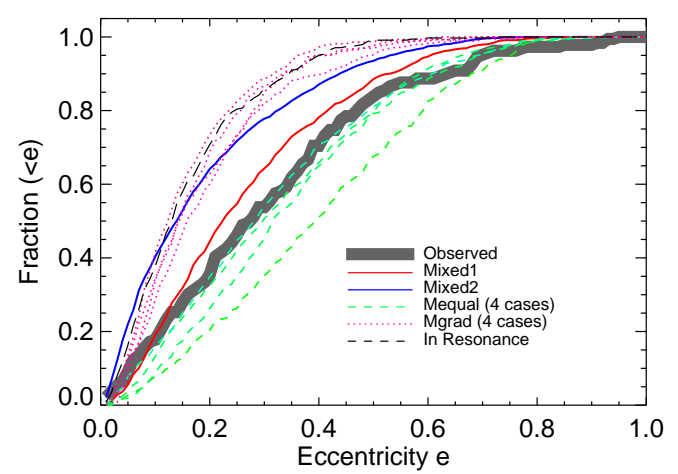

FIG. 1.- Cumulative eccentricity distribution of the known exoplanets beyond $0.1 \mathrm{AU}$ (thick grey), compared with our scattering simulations.

al.1996; Marzari \& Weidenschilling 2002). The outermost planet was placed two Hill radii interior to $10 \mathrm{AU}$; cases with more massive planets and therefore larger Hill radii therefore had the innermost planet closer to the star than for cases with lower-mass planets (see below). Planets were given zero eccentricity and mutual inclinations of less than 1 degree, and the stellar mass was $1 \mathrm{M}_{\odot}$.

We considered a range of planetary mass distributions. For our two largest sets (1000 simulations each) we randomly selected planet masses according to the observed distribution of exoplanet masses: $d N / d M \propto M^{-1.1}$ (Butler et al. 2006). In the "mixed1" set we restricted the planet mass $M_{p}$ to be between a Saturn mass $M_{\text {Sat }}$ and three Jupiter masses $M_{\text {Jup }}$. For our "mixed2" set, the minimum planet mass was decreased to $10 \mathrm{M}_{\oplus}$. We also performed four "Mequal" sets (500 simulations each) with equal mass planets for $M_{p}=30 \mathrm{M}_{\oplus}, M_{\text {Sat }}, M_{J u p}$, and $3 M_{\text {Jup }}$. Finally, the "Mgrad" sets (250 simulations each) contained radial gradients in $M_{p}$. For the JSN set, in order of increasing orbital distance, $M_{p}=M_{J u p}$, $M_{\text {Sat }}$, and $30 \mathrm{M}_{\oplus}$. For the NSJ set, these masses were reversed, i.e., the $M_{\text {Jup }}$ planet was the most distant. The 3JJS and SJ3J sets had, in increasing radial distance, $M_{p}$ $=3 M_{\text {Jup }}, M_{\text {Jup }}$ and $M_{\text {Sat }}$, and $M_{p}=M_{\text {Sat }}, M_{J u p}$ and $3 M_{\text {Jup }}$, respectively.

Each simulation was integrated with the hybrid version of the Mercury integrator (Chambers 1999). All planets were assigned physical densities of $1.3 \mathrm{~g} \mathrm{~cm}^{-3}$ and collisions were treated as inelastic mergers. We used a 20 day timestep which tests show introduces an error of less than 1 part in $10^{5}$ for perihelion distances larger than 0.5 $\mathrm{AU}$. In almost all cases energy was conserved to better than one part in $10^{4}$ for the entire $100 \mathrm{Myr}$ simulation, which Barnes \& Quinn (2004) showed is adequate precision to test stability. However, in some cases, energy was poorly conserved; those cases were rerun with a 5 day timestep. After this step, simulations with poor energy conservation were removed from the analysis.

\section{RESULTS}

Figure 1 shows that four of our sets of simulations match the exoplanet eccentricity distribution - mixed1, Mequal:Jup, Mequal:Sat, and Mequal:30 $\mathrm{M}_{\oplus}$ - with $\mathrm{P}$ values from K-S tests greater than 0.01 . However, given

of simulations were stable for $100 \mathrm{Myr}$ which shows that we started close to the stability boundary.

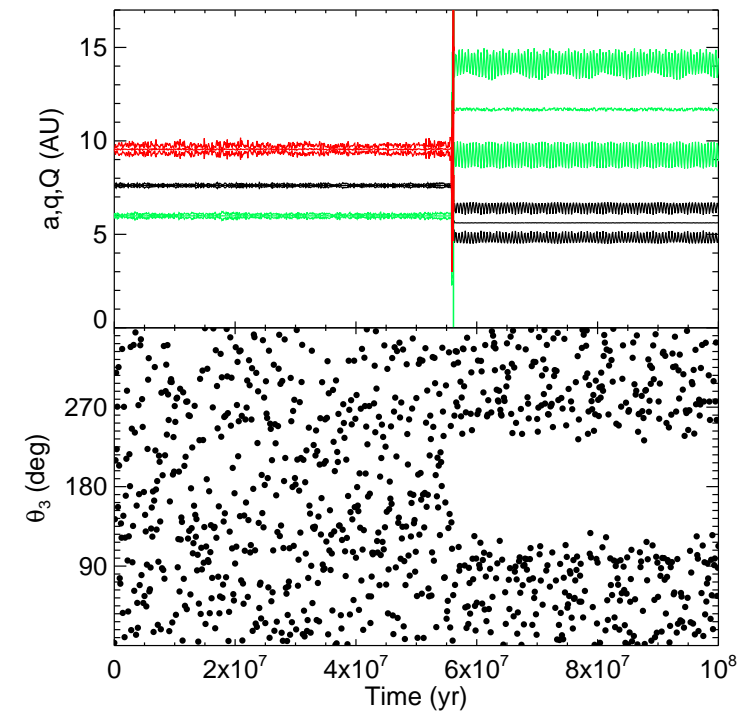

FIG. 2.- Evolution of a system that produced a pair of planets in the 3:1 MMR. Top: The three planets' semimajor axes $a$, perihelia $q$ and aphelia $Q$. The inner (green), middle (black), and outer (red) planets are 43, 105, and $16 \mathrm{M}_{\oplus}$, respectively. Bottom: Evolution of the $3: 1$ resonant argument $\theta_{3}=3 \lambda_{2}-\lambda_{1}-\left(\varpi_{1}+\varpi_{2}\right)$. Resonant libration starts immediately after ejection of the outer planet.

the increasing number of low-mass exoplanets, we believe that our mixed 1 and mixed2 simulations are the most realistic initial conditions. If scattering is the source of exoplanet eccentricities, then soon-to-be-discovered systems with lower-mass planets should indeed tend to have lower eccentricities (Ford \& Rasio 2008).

We found MMRs by examining resonant arguments for simulations which produced pairs of planets with period ratios close to commensurate values. A pair of planets is in resonance if any resonant argument $\theta_{i}$ librates rather than circulates. For MMR $\mathrm{p}+\mathrm{q}: \mathrm{p}$, arguments are of the form

$$
\theta_{i}=(p+q) \lambda_{1}-p \lambda_{2}-q \varpi_{1,2}
$$

where $\lambda$ are mean longitudes, $\varpi$ are longitudes of pericenter, and subscripts 1 and 2 refer to the inner and outer planet, respectively (e.g., Murray \& Dermott 1999).

Figure 2 shows the evolution of a typical simulation that created a resonant system. The instability started 55.8 Myr into the simulation, causing a series of close encounters. Within a few hundred thousand years the outer planet was ejected and the inner two planets swapped places. The two remaining planets are on stable orbits in the 3:1 MMR, and all three resonant arguments $\theta_{1,2,3}$ librate with amplitudes between $120^{\circ}$ and $160^{\circ}$.

A variety of MMRs is populated by scattering (see Table 2). Most common are the 2:1 and 3:1, but higherorder MMRs exist up to eleventh order (13:2). The resonant libration amplitudes tend to be large, with a median of $110^{\circ}$ and several cases with amplitudes of $\sim 170^{\circ}$. This contrasts with MMRs from migration which are created in a dissipative environment and should be much smaller. MMRs occur preferentially in cases with mixed mass distributions, especially those with a positive mass gradient such as Mgrad:NSJ. MMRs are relatively rare for equal mass planets, and they tend to arise more often after collisions rather than ejections, which contrasts with the mixed and Mgrad cases. This may explain why MMRs have not been found in previous studies (except for iso- 
TABLE 1

Candidate Resonant Planetary systems ${ }^{1}$

\begin{tabular}{c|c|c|c|c}
\hline \hline & \multicolumn{4}{c}{} \\
$\begin{array}{c}\text { System } \\
\text { (pair) }\end{array}$ & $\begin{array}{c}a_{1}, a_{2} \\
\text { (AU) }\end{array}$ & $e_{1}, e_{2}$ & $\begin{array}{c}M_{1}, M_{2} \\
\left(M_{J u p}\right)\end{array}$ & MMR \\
\hline & & & & \\
GJ 876 c-b & $0.13,0.2078$ & $0.27,0.025$ & $0.56,1.935$ & $2: 1$ \\
HD 73526 b-c & $0.66,1.05$ & $0.19,0.14$ & $2.9,2.5$ & $2: 1$ \\
HD 82943 c-b & $0.746,1.19$ & $0.359,0.219$ & $2.01,1.75$ & $2: 1$ \\
HD 128311 b-c & $1.099,1.76$ & $0.25,0.17$ & $2.18,3.21$ & $2: 1$ \\
$\mu$ Arae d-b & $0.921,1.497$ & $0.067,0.128$ & $0.522,1.676$ & $2: 1$ \\
GJ 317 b-c & $0.95,2.35$ & $0.19,0.42$ & $1.2,0.83$ & $4: 1^{2}$ \\
HD 108874 b-c & $1.051,2.68$ & $0.07,0.25$ & $1.36,1.018$ & $4: 1$ \\
HD 17156 b-c & $0.159,0.481$ & $0.6717,0.136$ & $3.111,0.063$ & $5: 1$ \\
HD 202206 b-c & $0.83,2.55$ & $0.435,0.267$ & $17.4,2.44$ & $5: 1$ \\
HD 208487 b-c & $0.49,1.8$ & $0.32,0.19$ & $0.45,0.46$ & $7: 1$ \\
& & & & \\
\hline
\end{tabular}

${ }^{a}$ See http://www.lpl.arizona.edu/ rory/research/xsp/dynamics/

${ }^{b}$ Johnson et al. (2007) did not determine apsidal angles, but Barnes $\&$ Greenberg (2008) used a stability analysis to predict that the system must be in the $4: 1 \mathrm{MMR}$.

lated cases in Adams \& Laughlin 2003 and Chatterjee et al. 2008).

MMRs appear to be populated at random: any stable region of parameter space can be accessed by scattering. To test this hypothesis, we calculated the phase space density of resonant orbits within $10 \%$ of the $2: 1$ and 3:1 MMRs for planetary mass ratio of $1 / 3,1$, and 3 , with $M_{\text {inner }}+M_{\text {outer }}=400 \mathrm{M}_{\oplus}$. For each MMR we ran $\sim 22,000$ 3-body (star + two planets) simulations for $1 \mathrm{Myr}$. The semimajor axis of the inner planet was fixed at $5 \mathrm{AU}$ (2:1 MMR) or $4 \mathrm{AU}$ (3:1 MMR). We sampled four parameters: the orbital period ratio, the inner and outer planets' eccentricities, and the relative apsidal orientation. Inclinations $\left(\right.$ of $<1^{\circ}$ ) and mean longitudes were sampled at random. Resonant orbits were found by libration of resonant arguments.

The density of 2:1 resonant orbits is higher for a more massive outer planet (Figure 3). ${ }^{6}$ Almost all of the 2:1 and 3:1 resonant orbits from scattering are found in areas of high resonant density, and near-resonant "false alarms" lie in areas of low density. Thus, scattering does indeed appear to populate MMRs at random. This explains why the 2:1 MMRs from our mixed1 and mixed2 simulations, with no initial mass gradients, have a median $M_{\text {inner }} / M_{\text {outer }}$ of 0.5 . In addition, the Mgrad:NSJ ( $\left.M_{\text {inner }} / M_{\text {outer }} \approx 1 / 3\right)$ systems formed a large number of 2:1 MMRs while the Mgrad:JSN $\left(M_{\text {inner }} / M_{\text {outer }} \approx 3\right)$ cases formed far fewer.

For the parameter space we sampled, the integrated 3:1 resonant density is $\sim 40 \%$ less than the $2: 1$ density. However, the available parameter space is not evenly populated by scattering. For example, scattering causes more massive planets to be closer to the star (Chatterjee et al. 2008). Indeed, the integrated $2: 1$ resonant density for $M_{\text {inner }} / M_{\text {outer }}=1 / 3$ is $66 \%$ higher than the 3:1 density. This explains the increased number of 2:1 vs. 3:1 MMRs in our sample - 74 cases of the 2:1 MMR and 47 of the $3: 1(61 \%$ more in $2: 1)$.

The density of resonant orbits can explain other features of the population of resonant planets. The resonant planets tend to have smaller eccentricities than the

6 For a more detailed study of the 2:1 MMR, see Marzari et al. (2006) and Michtchenko et al. (2008a, 2008b).

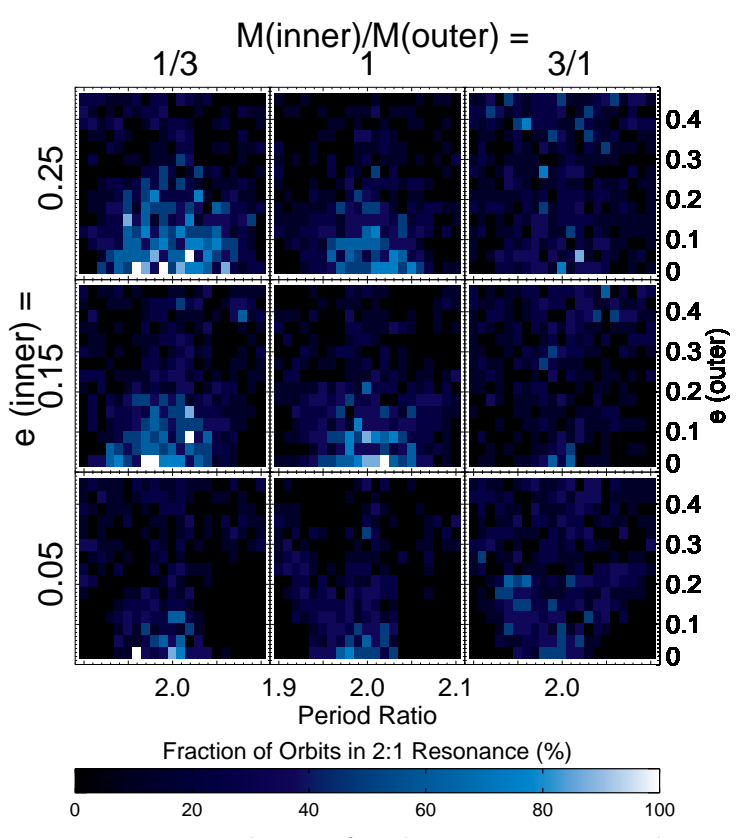

FIG. 3.- Resonant density for the 2:1 MMR. For these simulations, the inner planet's semimajor axis was fixed at $5 \mathrm{AU}$, and its eccentricity was varied between 0.05 and 0.25 . The color corresponds to the fraction of orbits in resonance for a given value of the outer planet's semimajor axis and eccentricity (see color bar), averaged over 8 simulations with different apsidal alignments.

non-resonant planets (Fig. 1). Indeed, resonant systems underwent an average of about five times fewer close encounters between planets before the system stabilized ( $30-50$ vs. $100->200$ encounters), compared with the median outcome. The time between the first instability and stabilization for resonant cases was $\sim 50,000$ years, a factor of about five shorter than for the non-resonant systems.

Low-order MMRs preferentially arise in systems containing a planet with a large Safronov number $S$. $S$ is the ratio of the escape speed from a planet's surface to the escape speed from the system, $S=$ $\left(M_{p} / M_{\star}\right)^{1 / 2}\left(a_{p} / R_{p}\right)^{1 / 2}$, where $M_{\star}$ is the stellar mass, $a_{p}$ and $R_{p}$ are the planet's orbital distance and radius, respectively (Safronov 1969). Planets with larger $S$ give stronger velocity kicks and thereby reduce the number of encounters needed to eject a planet. Indeed, the $2: 1$ and 3:1 MMRs correlate with systems with at least one planetary $S$ value above 4 . In the mixed 2 set, which is the only set with a significant range in $S$, the median $S_{\max }$ for the 2:1 and 3:1 MMRs is 4.5, as compared with 3.5 for all unstable mixed2 simulations. A K-S test shows that the two samples are indeed different at the $99.9 \%$ confidence level. This constrains where the $2: 1$ or $3: 1$ MMRs can arise as a function of $M_{p}, M_{\star}, a_{p}$, and $R_{p}$ : only systems with relatively high-mass planets $\left(M_{p} \gtrsim M_{J u p}\right)$ can generate these MMRs close-in. In contrast, high-order (4:1 and higher) MMRs from the mixed2 set match the sample of unstable cases and so are not constrained.

The MMRs we found are numerically robust. The median fractional integration error $\mathrm{dE} / \mathrm{E}$ for the 170 resonant systems was $2.6 \times 10^{-8}$, far below the $\sim 10^{-4}$ limit for determining stability (Barnes \& Quinn 2004), and smaller than the median $\mathrm{dE} / \mathrm{E}$ of $1.2 \times 10^{-7}$ for all unstable systems. MMRs tend to arise in cases with short encounter times and relatively low final eccentric- 
TABLE 2

RESONANCES FROM SCATTERING SIMULATIONS

\begin{tabular}{|c|c|c|c|}
\hline Set & $\begin{array}{l}\text { Nsims - } \\
\text { unstable(\%) }\end{array}$ & $\begin{array}{l}\mathrm{N}(\%) \text { in } \\
\text { MMRs }\end{array}$ & $\begin{array}{l}\text { MMRs } \\
(\%)\end{array}$ \\
\hline Mixed1 & $965-569(59 \%)$ & $27(4.7 \%)$ & $\begin{array}{ll}2: 1(1.6 \%), & 3: 1 \\
(1.6 \%), & 4: 1 \\
(0.7 \%), & 5: 1, \\
6: 1,7: 2,9: 2\end{array}$ \\
\hline Mixed2 & $982-744(76 \%)$ & $52(7 \%)$ & 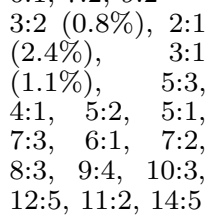 \\
\hline Mequal: $3 M_{J}$ & $368-241(65 \%)$ & $1(0.4 \%)$ & $7: 1$ \\
\hline Mequal: $M_{J}$ & $452-232(51 \%)$ & $4(1.7 \%)$ & $\begin{array}{l}2: 1, \quad 3: 1, \quad 4: 1 \\
(0.9 \%)\end{array}$ \\
\hline Mequal: $M_{S a t}$ & $390-362(93 \%)$ & $14(3.9 \%)$ & $\begin{array}{l}2: 1(1.9 \%), 3: 1 \\
(0.6 \%), 5: 2,5: 1 \\
(0.6 \%), 6: 1,7: 1\end{array}$ \\
\hline Mequal:30 $\mathrm{M}_{\oplus}$ & $367-365(99 \%)$ & $10(2.7 \%)$ & $\begin{array}{l}2: 1(1.9 \%), 3: 1, \\
11: 6\end{array}$ \\
\hline Mgrad:JSN & $250-206(82 \%)$ & $13(6.3 \%)$ & $\begin{array}{ll}2: 1 \quad(1 \%), & 3: 1 \\
(1.9 \%), & 4: 1, \\
5: 2, \quad 5: 1, & 7: 3, \\
8: 3,13: 2 & \end{array}$ \\
\hline Mgrad:NSJ & $245-221(90 \%)$ & $30(14.6 \%)$ & $\begin{array}{l}2: 1(9.2 \%), 3: 1 \\
(2.3 \%), \quad 5: 2, \\
7: 3,7: 2,11: 5\end{array}$ \\
\hline Mgrad:3JJS & $250-150(60 \%)$ & $4(2.7 \%)$ & $\begin{array}{l}3: 1,4: 1 \text { (1.3\%), } \\
11: 3\end{array}$ \\
\hline Mgrad:SJ3J & $245-219(89 \%)$ & $16(6.5 \%)$ & $3: 1(5.7 \%), 4: 1$ \\
\hline
\end{tabular}

ities. Those situations yield smaller $\mathrm{dE} / \mathrm{E}$ than for the more common stronger encounters that lead to very eccentric planets. Thus, our cutoff of $\mathrm{dE} / \mathrm{E}<10^{-4}$ allows us to accurately sample both eccentricities and MMRs.

\section{DISCUSSION AND CONCLUSIONS}

Planet-planet scattering creates MMRs. The typical path to a resonant system involves several close encounters between one smaller and two larger planets. After a relatively short time of instability, the smaller planet is destroyed, usually via ejection ( $78 \%$ of all cases) or collision $(22 \%)$, leaving behind a pair of resonant planets. Relatively weak instabilities are probably very common in planetary systems; one may even have occurred in our own Solar System (Thommes et al. 1999). Nonetheless, only a fraction of unstable systems produce resonant planets, typically $5-10 \%$. These systems have large libration amplitudes and occupy a range of low-and highorder MMRs (Table 2). Most of these resonances are indefinitely stable; we integrated the 170 resonant systems for an additional 1 Gyr and only 9 (5\%) left the resonance, 4 cases leading to an additional system instability.

It may be possible to tell apart resonant exoplanets created via scattering from those created via convergent migration. In fact, only one resonant system appears to be inconsistent with a scattering origin due to its very low-amplitude libration (GJ 876; Marcy et al. 2001). If two planets are trapped in the $2: 1$ or $3: 2 \mathrm{MMR}$ and the inner planet is the more massive, then migration can be stopped or even reversed (Masset \& Snellgrove 2001; Crida \& Morbidelli 2007). However, if the outer planet is the more massive then inward migration continues. In contrast, scattering produces planets in a variety of MMRs (including the 3:2 and 2:1) with a wide range in mass ratios and a preference for the outer planet to be more massive. Thus, scattering is likely to be responsible for systems past $\sim 1$ AU with 2:1 or 3:2 resonant planets and a more massive outer planet. The HD 128311 and $\mu$ Arae systems are good candidates for creation via scattering (Table 1; see also Sándor \& Kley 2006).

Several extra-solar systems show tentative evidence for high-order MMRs - 4:1, 5:1 and even 7:1 (Table 1; Johnson et al. 2007; Correia et al. 2005; Gregory 2007; Short et al. 2008). No study to date has shown that migration could capture planets in MMRs of higher order than $2: 1$, although we encourage expanded studies of this process. $^{7}$ Scattering produces a wide range of high-order resonances (Table 2). Thus, if the current candidate high-order MMR systems are confirmed (Table 1), then scattering is likely to be the responsible mechanism.

Turbulence in gaseous disks may destroy MMRs, leaving perhaps only $\sim 1 \%$ of planet pairs in resonance (Adams et al.2008). This effect is stronger for higherorder MMRs. However, the timescale for MMR destruction is sensitive to the strength of MRI turbulence which is very uncertain. In particular, if the MRI is fully or partially suppressed by the low ionization fraction in the inner protoplanetary disk (Gammie 1996) then the survival prospects for resonant planets would be improved. Nonetheless, if only a few percent of systems remain in resonance, then scattering and migration may provide a comparable number of MMRs.

Our simulations do not account for any dissipation. However, instabilities may occur while some gas remains in the disk (Moeckel et al. 2008). In that case, MMRs could still result from scattering (Lee et al. 2008) and perhaps have smaller libration amplitudes.

In conclusion, we have identified a new mechanism for the creation of exoplanet systems in MMRs. Unfortunately the current data do not allow a conclusive determination of a resonance, let alone precise descriptions of the resonant argument oscillation. Nonetheless, our scattering model has important distinctions from the convergent migration model: high-order MMRs, largeamplitude resonant libration, and low-order MMRs with $M_{\text {inner }} / M_{\text {outer }}<1$. As the orbital properties of exoplanets are better determined, it should be possible to distinguish between these scenarios.

We thank Google for access to their machines. We are grateful to Greg Laughlin, Dimitri Veras, and an anonymous referee for helpful input. S.N.R. was supported by the NASA Postdoctoral Program administered by Oak Ridge Associated Universities through a contract with NASA. R.B. acknowledges support from NASA's PG\&G grant NNG05GH65G and NASA Terrestrial Planet Finder Foundation Science grant 811073.02.07.01.15. 
7 Highly-damped bodies can undergo resonant shepherding by the 6:1 or even 8:1 MMRs (Raymond et al.2006; Mandell et al. 2007). However, as bodies grow the damping decreases, and shepherded planets do not survive in resonance.

\section{REFERENCES}

Adams, F. C., \& Laughlin, G. 2003, Icarus, 163, 290

Adams, F. C., Laughlin, G., \& Bloch, A. M. 2008, ApJ, 683, 1117

Barnes, R., \& Greenberg, R. 2008, IAU Symposium, 249, 469

Barnes, R., \& Quinn, T. 2004, ApJ, 611, 494

Butler, R. P., et al. 2006, ApJ, 646, 505

Chambers, J. E. 1999, MNRAS, 304, 793

Chatterjee, S., Ford, E. B., Matsumura, S., \& Rasio, F. A. 2007, ArXiv Astrophysics e-prints, arXiv:astro-ph/0703166

Correia, A. C. M., Udry, S., Mayor, M., Laskar, J., Naet, D., Pepe, F., Queloz, D., \& Santos, N. C. 2005, A\&A, 440, 751

Crida, A., \& Morbidelli, A. 2007, MNRAS, 377, 1324

Ford, E. B., \& Rasio, F. A. 2007, ArXiv Astrophysics e-prints, arXiv:astro-ph/0703163

Gammie, C. F. 1996, ApJ, 457, 355

Gregory, P. C. 2007, MNRAS, 374, 1321

Jackson, B., Greenberg, R., \& Barnes, R. 2008, ApJ, 678, 1396

Johnson, J. A., Butler, R. P., Marcy, G. W., Fischer, D. A., Vogt, S. S., Wright, J. T., \& Peek, K. M. G. 2007, ApJ, 670, 833

Juric, M., \& Tremaine, S. 2007, ArXiv Astrophysics e-prints, arXiv:astro-ph/0703160

Lee, A. T., Thommes, E. W., \& Rasio, F. A. 2008, ArXiv e-prints, 801, arXiv:0801.1926

Lin, D. N. C., \& Ida, S. 1997, ApJ, 477, 781

Mandell, A. M., Raymond, S. N., \& Sigurdsson, S. 2007, ApJ, 660, 823

Marcy, G. W., Butler, R. P., Fischer, D., Vogt, S. S., Lissauer, J. J., \& Rivera, E. J. 2001, ApJ, 556, 296

Marzari, F., \& Weidenschilling, S. J. 2002, Icarus, 156, 570
Marzari, F., Scholl, H., \& Tricarico, P. 2006, A\&A, 453, 341

Masset, F., \& Snellgrove, M. 2001, MNRAS, 320, L55

Michtchenko, T. A., Beaugé, C., \& Ferraz-Mello, S. 2008a, MNRAS, 387, 747

Michtchenko, T. A., Beaugé, C., \& Ferraz-Mello, S. 2008b, MNRAS, in press.

Moeckel, N., Raymond, S. N., \& Armitage, P. J. 2008, ArXiv eprints, 807, arXiv:0807.4186

Pierens, A., \& Nelson, R. P. 2008, A\&A, 482, 333

Pollack, J. B., Hubickyj, O., Bodenheimer, P., Lissauer, J. J., Podolak, M., \& Greenzweig, Y. 1996, Icarus, 124, 62

Rasio, F. A., Tout, C. A., Lubow, S. H., \& Livio, M. 1996, ApJ, 470, 1187

Rasio, F. A., \& Ford, E. B. 1996, Science, 274, 954

Raymond, S. N., Mandell, A. M., \& Sigurdsson, S. 2006, Science, 313,1413

Safronov, V. S. 1969, 1969.

Sándor, Z., \& Kley, W. 2006, A\&A, 451, L31

Short, D., Welsh, W. F., Orosz, J. A., \& Windmiller, G. 2008, ArXiv e-prints, 803, arXiv:0803.2935

Snellgrove, M. D., Papaloizou, J. C. B., \& Nelson, R. P. 2001, A\&A, 374,1092

Thommes, E. W. 2005, ApJ, 626, 1033

Thommes, E. W., Duncan, M. J., \& Levison, H. F. 1999, Nature, 402,635

Weidenschilling, S. J., \& Marzari, F. 1996, Nature, 384, 619 